\title{
A REGULARITY THEOREM FOR COMPOSITE FUNCTIONAL EQUATIONS
}

\author{
ATTILA GILÁNYI AND ZSOLT PÁLES
}

ABSTRACT. In this paper a regularity theorem for the functional equation

$$
\begin{aligned}
f(x+y)-f(x) & +\sum_{i=1}^{n} \varphi_{i}\left[g_{i}\left(y+z_{i}\right)-g_{i}(y)\right] \\
& =\sum_{i=1}^{n} \psi_{i}\left[g_{i}\left(y+x+z_{i}\right)-g_{i}(y+x)-g_{i}\left(y+z_{i}\right)+g_{i}(y)\right]
\end{aligned}
$$

is proved.

In the regularity theory of functional equations, problems are considered, where the unknown functions have "weak" regularity properties (monotonicity, measurability, etc.) and a "stronger" regularity (e.g. differentiability) is proved. The most general and significant results in this field are due to A. Járai. (cf. e.g. [8], [9], [10]; [6], [11], [12]). In the cited papers functional equations are studied in which the unknown functions are not substituted into each other. Regularity properties of composite functional equations, motivated by a problem of J. Aczél [1], were first studied by Zs. Páles [16], [17]. Developing these ideas and methods, in [18] and [19], the functional equation

$$
\begin{array}{r}
f(x+y)-f(x)+\varphi(g(y+z)-g(y))=\psi(g(x+y+z)-g(y+z)-g(x+y)+g(y)) \\
(x, y, z>0, x+y+z<\alpha)
\end{array}
$$

was considered, where $0<\alpha \leq \infty, f, g: I \rightarrow \mathbb{R}, \varphi: J \rightarrow \mathbb{R}, \psi: H \rightarrow \mathbb{R}$ and $I=] 0, \alpha[, \quad J=\{g(y+z)-g(y) \mid y, z>0, y+z<\alpha\}, H=\{g(y+x+z)-g(y+$ $x)-g(y+z)+g(y) \mid x, y, z>0, y+x+z<\alpha\}$, and it was shown that the strict monotonicity of the solutions $f, g, \varphi, \psi$ of the equation above implies that $f$ and $g$ are strictly convex or strictly concave, differentiable functions, the sets $J$ and $H$ are open intervals and the functions $\varphi$ and $\psi$ are also differentiable. In [18] and [19] the functional equation above was also completely solved. A crucial step of the argument therein was to obtain the differentiability properties of the unknown functions. Then differentiating the equation with respect to the variables, one can eliminate the subexpressions containing composite functions. The functional equation so obtained can be handled with common and general methods of the theory of noncomposite functional equations.

Mathematics Subject Classification (2000): 39B22, 26A48, 26 A51.

Keywords and phrases: composite functional equations, regularity.

Research supported by the Hungarian National Foundation for Scientific Research (OTKA) Grant T-030082, by the Hungarian Higher Education, Research, and Development Found (FKFP) Grant 0310/1997, and, partially, by the German Academic Exchange Service (DAAD). 
In the present paper, extending the methods used in [18] and [19], we generalize the regularity result above for a broad class of functional equations. Our investigation has been motivated by some recent applications of functional equations of this type in social and behavioral sciences (c.f. [2], [3], [4], [14] and [15]). Some of the problems dealt with in these papers were reduced to functional equations that are special cases of the equation treated in our main theorem. Therefore, this theorem can be useful to determine the solutions of similar concrete equations. The result given here was also announced during the $7^{\text {th }}$ International Conference on Functional Equations and Inequalities, Złockie, Poland, 1999 (cf. [7]).

Our result reads as follows.

Theorem. Let us consider the functional equation

$$
\begin{aligned}
& f(x+y)-f(x)+ \sum_{i=1}^{n} \varphi_{i}\left[g_{i}\left(y+z_{i}\right)-g_{i}(y)\right] \\
&= \sum_{i=1}^{n} \psi_{i}\left[g_{i}\left(y+x+z_{i}\right)-g_{i}(y+x)-g_{i}\left(y+z_{i}\right)+g_{i}(y)\right] \\
& \quad\left(x, y, z_{i}>0, x+y+z_{i}<\alpha ; i=1, \ldots, n\right),
\end{aligned}
$$

where $n$ is a positive integer, $0<\alpha \leq \infty, f, g_{i}: I \rightarrow \mathbb{R}, \varphi_{i}: J_{i} \rightarrow \mathbb{R}, \psi_{i}: H_{i} \rightarrow \mathbb{R}$ and

$$
\begin{aligned}
I & =] 0, \alpha[ \\
J_{i} & =\left\{g_{i}\left(y+z_{i}\right)-g_{i}(y) \mid y, z_{i}>0, y+z_{i}<\alpha\right\}, \\
H_{i} & =\left\{g_{i}\left(y+x+z_{i}\right)-g_{i}(y+x)-g_{i}\left(y+z_{i}\right)+g_{i}(y) \mid x, y, z_{i}>0, y+x+z_{i}<\alpha\right\},
\end{aligned}
$$

for $i=1, \ldots, n$. Suppose, that the functions $\varphi_{1}, \ldots, \varphi_{n}, \psi_{1}, \ldots, \psi_{n}, g_{1}, \ldots, g_{n}$ and $f$ satisfy (1), furthermore, $\varphi_{1}, \ldots, \varphi_{n} ; \psi_{1}, \ldots, \psi_{n}$ and $g_{1}, \ldots, g_{n}$ are strictly monotonic, and the elements of each n-tuple in the same way.

Then

- $f$ is strictly convex or strictly concave and continuously differentiable on I;

- $g_{1}, \ldots, g_{n}$ are all strictly convex or strictly concave on $I$;

- $J_{1}, \ldots, J_{n}$ and $H_{1}, \ldots, H_{n}$ are open intervals;

$-\varphi_{1}, \ldots, \varphi_{n}$ and $\psi_{1}, \ldots, \psi_{n}$ are differentiable on $J_{1}, \ldots, J_{n}$ and $H_{1}, \ldots, H_{n}$, respectively.

Proof. First we prove that the functions $g_{i},(i=1, \ldots, n)$ are all strictly convex or strictly concave. Because of the symmetry of equation (1), it is enough to prove the statement for $i=1$. If we fix all the variables except for $z_{1}$ in (1), by the strict monotonicity of $\varphi_{1}$ and $g_{1}$ we get, that the left hand side of (1) is a strictly monotonic function of $z_{1}$. Therefore, the right hand side is strictly monotonic, too, so, for fixed $\bar{x}, \bar{y}>0, \bar{x}+\bar{y}<\alpha$, the function

$$
z \rightarrow g_{1}(\bar{x}+\bar{y}+z)-g_{1}(\bar{y}+z) \quad(z \in] 0, \alpha-\bar{x}-\bar{y}[),
$$

that is, $\left.G_{1}:\right] 0, \alpha-\bar{x}[\rightarrow \mathbb{R}$,

$$
G_{1}(z)=g_{1}(\bar{x}+z)-g_{1}(z) \quad(z \in] 0, \alpha-\bar{x}[),
$$


is also strictly monotonic. (Moreover, the functions $\left.G_{i}:\right] 0, \alpha-\bar{x}[\rightarrow \mathbb{R}$,

$$
G_{i}(z)=g_{i}(\bar{x}+z)-g_{i}(z) \quad(z \in] 0, \alpha-\bar{x}[)
$$

are strictly monotonic in the same way for each $i \in\{1, \ldots, n\}$.) Let us suppose that $G_{1}$ is strictly monotone increasing. We have

$$
\begin{aligned}
g_{1}\left(\frac{s+t}{2}\right)-g_{1}(s) & <g_{1}\left(s+\frac{t-s}{2}+\frac{t-s}{2}\right)-g_{1}\left(s+\frac{t-s}{2}\right) \\
& =g_{1}(t)-g_{1}\left(\frac{s+t}{2}\right)
\end{aligned}
$$

for arbitrary $s, t \in] 0, \alpha\left[, s<t\right.$, which gives the strict Jensen convexity of $g_{1}$. By the theorem of Bernstein-Doetsch [5] (c.f. also [13], Chapter VI, §4), this fact and the monotonicity of $g_{1}$ implies the strict convexity of $g_{1}$. Obviously, if $G_{1}$ is strictly monotone decreasing then we get that $g_{1}$ is strictly concave. The functions $G_{i}$ defined in (2) are strictly monotonic in the same way, thus, $g_{1}, \ldots, g_{n}$ are all strictly convex or all strictly concave.

Now we show the strict convexity or concavity of $f$. Since $g_{1}, \ldots, g_{n}$ are strictly convex or concave, for fixed $\bar{y}, \bar{z}>0, \bar{y}+\bar{z}<\alpha$, the functions

$$
x \rightarrow g_{i}(x+\bar{y}+\bar{z})-g_{i}(x+\bar{y}) \quad(x \in] 0, \alpha-\bar{y}-\bar{z}[)
$$

are strictly monotonic in the same way for $i \in\{1, \ldots, n\}$. The functions $\psi_{1}, \ldots, \psi_{n}$ are also strictly monotonic in the same way, thus, the left hand side of (1) is a strictly monotonic function of $x$, that is,

$$
x \rightarrow f(x+\bar{y})-f(x) \quad(x \in] 0, \alpha-\bar{y}[)
$$

is strictly monotonic. Using a similar method as above, we get the strict Jensen convexity or concavity of $f$, which, together with the theorem of Bernstein-Doetsch, yields the strict convexity or concavity of $f$.

Thus, the functions $f, g_{1}, \ldots, g_{n}: I \rightarrow \mathbb{R}$ are strictly convex or concave. Therefore, they are also continuous, there exists their left and right derivative at every point $x \in I$, moreover, they are differentiable everywhere but (at most) at countably many points in $I$. Since these functions are strictly monotonic, they are invertible, and their inverses also have the properties above (Cf. [13], Chapter VII.)

The continuity of $g_{1}, \ldots, g_{n}$ gives that $J_{1}, \ldots, J_{n}$ and $H_{1}, \ldots, H_{n}$ are intervals, the strict monotonicity and the strict convexity or concavity of $g_{1}, \ldots, g_{n}$ implies that these intervals are open.

In the next step we prove that the function $\varphi_{i}$ is differentiable on $J_{i}$ for $i=1, \ldots, n$. Due to symmetry, we may assume that $i=1$. For a $\bar{u} \in J_{1}$, there exist $\bar{x}, \bar{y}, \bar{z}_{1}>0$ such that $\bar{x}+\bar{y}+\bar{z}_{1}<\alpha$ and $\bar{u}=g_{1}\left(\bar{y}+\bar{z}_{1}\right)-g_{1}(\bar{y})$. Thus, $\bar{z}_{1}=g_{1}^{-1}\left(\bar{u}+g_{1}(\bar{y})\right)-\bar{y}$ and the function $(u, y) \rightarrow g_{1}^{-1}\left(u+g_{1}(y)\right)-y$ is defined in a neighbourhood of $(\bar{u}, \bar{y})$. Writing $g_{1}^{-1}\left(u+g_{1}(y)\right)-y$ for $z_{1}$ in $(1)$ we get that, for fixed $\bar{z}_{2}, \ldots, \bar{z}_{n}$, there exist 
some neighbourhoods $U, V, W$ of $\bar{u}, \bar{x}, \bar{y}$, respectively, such that

$$
\begin{aligned}
\varphi_{1}(u)= & f(x)-f(x+y) \\
& -\sum_{i=2}^{n} \varphi_{i}\left(g_{i}\left(y+\bar{z}_{i}\right)-g_{i}(y)\right) \\
& +\psi_{1}\left[g_{1}\left(x+g_{1}^{-1}\left(u+g_{1}(y)\right)\right)-u-g_{1}(y+x)\right] \\
& +\sum_{i=2}^{n} \psi_{i}\left(g_{i}\left(y+x+\bar{z}_{i}\right)-g_{i}(y+x)-g_{i}\left(y+\bar{z}_{i}\right)+g_{j}(y)\right)
\end{aligned}
$$

holds for $(u, x, y) \in U \times V \times W$. We show that there exist $x \in V$ and $y \in W$ such that the right hand side of (3) is differentiable at $\bar{u}$ with respect to $u$. Let us denote the sets, where $g_{1}, g_{1}^{-1}$ and $\psi_{1}$ are not differentiable, by $A, B$ and $C$, respectively. Since $g_{1}$ and $g_{1}^{-1}$ are strictly convex or concave, the sets $A$ and $B$ are countable, the strict monotonicity of $\psi_{1}$ and Lebesgue's theorem imply that the Lebesgue measure of $C$ is 0 . Because of the strict monotonicity of $g_{1}$, the set of the points $y \in W$, for which $\bar{u}+g_{1}(y) \in B$, is countable. Thus, there exists a $y^{*} \in W$, such that the function $u \rightarrow g_{1}^{-1}\left(u+g_{1}\left(y^{*}\right)\right)$ is differentiable at $\bar{u}$ with respect to $u$. Obviously, the set $L \subset V$

$$
L=\left\{x \in V \mid x+g_{1}^{-1}\left(\bar{u}+g_{1}\left(y^{*}\right)\right) \in A\right\}
$$

is countable, so, for each (fixed) $x \in V \backslash L$ the function $u \rightarrow g_{1}\left(x+g_{1}^{-1}\left(\bar{u}+g_{1}\left(y^{*}\right)\right)\right)$ is differentiable at $\bar{u}$ with respect to $u$. The strict convexity or concavity of $g_{1}$ and $\bar{u} \neq 0$ imply that the function $G_{1}: V \rightarrow \mathbb{R}$,

$$
G_{1}(x)=g_{1}\left(x+g_{1}^{-1}\left(\bar{u}+g_{1}\left(y^{*}\right)\right)\right)-\bar{u}-g_{1}\left(x+y^{*}\right) \quad(x \in V)
$$

is strictly monotonic in $V$. Therefore, $G_{1}(V \backslash L)=G_{1}(V) \backslash G_{1}(L)$ and $G_{1}(V)$ is open, so, $G_{1}(V \backslash L)$ has a positive measure. Thus, there exists an $x^{*} \in V \backslash L$ with the property $G_{1}\left(x^{*}\right) \notin C$. For this element, the function

$$
u \rightarrow \psi_{1}\left(g_{1}\left(x^{*}+g_{1}^{-1}\left(u+g_{1}\left(y^{*}\right)\right)\right)-u-g_{1}\left(x^{*}+y^{*}\right)\right)
$$

is differentiable at $\bar{u}$ with respect to $u$, which implies that the right hand side of (3) is differentiable at $\bar{u}$ with respect to $u$, therefore, $\varphi_{1}$ also has this property.

We show the differentiability of $\psi_{i}$ on $H_{i}(i=1, \ldots, n)$. Similarly as above, we consider only the case $i=1$. For a fixed $\bar{u} \in H_{i}$, there exist $\bar{x}, \bar{y}, \bar{z}_{1}>0$ satisfying $\bar{x}+\bar{y}+\bar{z}_{1}<\alpha$ and

$$
\bar{u}=g_{1}\left(\bar{x}+\bar{y}+\bar{z}_{1}\right)-g_{1}(\bar{x}+\bar{y})-g_{1}\left(\bar{y}+\bar{z}_{1}\right)-g_{1}(\bar{y}) .
$$

The strict convexity or concavity of $g_{1}$ implies that the function

$$
G_{1}(s)=g_{1}(\bar{x}+s)-g_{1}(s) \quad(s \in] 0, \alpha-\bar{x}[
$$


is strictly monotonic and continuous. Writing $\bar{x}=x$ and $s=y+z_{1}$ in (1), we get

$$
\begin{aligned}
& f(\bar{x}+y)-f(\bar{x})+\varphi_{1}\left[g_{1}\left(y+z_{1}\right)-g_{1}(y)\right]+\sum_{i=2}^{n} \varphi_{i}\left[g_{i}\left(y+z_{i}\right)-g_{i}(y)\right] \\
& =\psi_{1}\left[g_{1}(\bar{x}+s)-g_{1}(s)-g_{1}(\bar{x}+y)+g_{1}(y)\right] \\
& \quad+\sum_{i=2}^{n} \psi_{i}\left[g_{i}\left(y+\bar{x}+z_{i}\right)-g_{i}(y+\bar{x})-g_{i}\left(y+z_{i}\right)+g_{i}(y)\right]
\end{aligned}
$$

that is,

$$
\begin{aligned}
& f(\bar{x}+y)-f(\bar{x})+\varphi_{1}\left[g_{1}(s)-g_{1}(y)\right]+\sum_{i=2}^{n} \varphi_{i}\left[g_{i}\left(y+z_{i}\right)-g_{i}(y)\right] \\
& =\psi_{1}\left[G_{1}(s)-G_{1}(y)\right] \\
& \quad+\sum_{i=2}^{n} \psi_{i}\left[g_{i}\left(y+\bar{x}+z_{i}\right)-g_{i}(y+\bar{x})-g_{i}\left(y+z_{i}\right)+g_{i}(y)\right],
\end{aligned}
$$

for $y, s \in] 0, \alpha-\bar{x}\left[, s>y\right.$ and $x, y, z_{i}>0, x+y+z_{i}<\alpha(i=2, \ldots, n)$. For $u=$ $G_{1}(s)-G_{1}(y)$, we have $s=G_{1}^{-1}\left(G_{1}(y)+u\right)$ and, by (4) and $(5), \bar{u}=G_{1}(\bar{y}+\bar{z})-G_{1}(\bar{y})$. Therefore, (3) implies that, for fixed $\bar{z}_{1}, \ldots, \bar{z}_{n}$,

$$
\begin{array}{ll}
\psi_{1}(u)= & f(\bar{x}+y)-f\left(\bar{x}+\varphi\left[g_{1}\left(G_{1}^{-1}\left(G_{1}(y)+u\right)\right)-g_{1}(y)\right]+\sum_{i=2}^{n} \varphi_{i}\left[g_{i}\left(y+\bar{z}_{i}\right)-g_{i}(y)\right]\right. \\
(7) & -\sum_{i=2}^{n} \psi_{i}\left[g_{i}\left(y+\bar{x}+\bar{z}_{i}\right)-g_{i}(y+\bar{x})-g_{i}\left(y+\bar{z}_{i}\right)+g_{i}(y)\right]((u, y) \in U \times V)
\end{array}
$$

in some neighbourhoods $U$ and $V$ of $\bar{u}$ and $\bar{y}$. The set of the points, where $g_{1}$ is not differentiable, is countable. Therefore, by the strict monotonicity of $G_{1}$ and $G_{1}^{-1}$, there exists a $y^{*} \in V$, such that $G_{1}^{-1}$ is differentiable at $G_{1}\left(y^{*}\right)+\bar{u}$ and $g_{1}$ is differentiable at $G_{1}^{-1}\left(G_{1}\left(y^{*}\right)+\bar{u}\right)$ with respect to $u$. Thus, the right hand side of (7) is differentiable at $\bar{u}$ with respect to $u$, which implies the same property for $\psi_{1}$.

Finally, we prove the differentiability of $f$ on $I$. Equation (1) implies that, for fixed $\bar{x}, \bar{y}, \bar{z}_{1}, \ldots, \bar{z}_{n}>0$ satisfying $\bar{x}+\bar{y}+\bar{z}_{i}<\alpha,(i=1, \ldots, n)$, there exist some neighbourhoods $V, W, Z_{i}$ of $\bar{x}, \bar{y}, \bar{z}_{i},(i=1, \ldots, n)$, respectively, such that

$$
\begin{aligned}
& f(x)= f(x+y)+\sum_{i=1}^{n} \varphi_{i}\left[g_{i}\left(y+z_{i}\right)-g_{i}(y)\right] \\
&-\sum_{i=1}^{n} \psi_{i}\left[g_{i}\left(y+x+z_{i}\right)-g_{i}(y+x)-g_{i}\left(y+z_{i}\right)+g_{i}(y)\right] \\
& \quad\left(\left(x, y, z_{i}\right) \in V \times W \times Z_{i} ; i=1, \ldots, n\right) .
\end{aligned}
$$

The functions $g_{1}, \ldots, g_{n}$ and $f$ are differentiable everywhere but at countably many points, therefore, there exists a $y^{*} \in W$ such that $g_{1}, \ldots, g_{n}$ and $f$ are differentiable at $\bar{x}+y^{*}$ with respect to $x$. Similarly, there exists a $z_{i}^{*} \in Z_{i}$ such that $g_{i}$ is differentiable at $y^{*}+\bar{x}+z_{i}^{*}$ for each $i \in\{1, \ldots, n\}$. The functions $\psi_{1}, \ldots, \psi_{n}$ are differentiable, thus, 
the right hand side of (8) is differentiable at $\bar{x}$ with respect to $x$, which implies the differentiability of $f$ at $\bar{x}$. The convexity or concavity and the differentiability of $f$ yield that $f$ is continuously differentiable on $I$.

\section{REFERENCES}

[1] J. Aczél, Problem 3, Report of Meeting, Aequationes Math. 56(1998), 299.

[2] J. Aczél, Gy. Maksa, C.T. Ng, Zs. Páles, A functional equation arising from ranked additive and separable utility, to appear in Proc. Amer. Math. Soc.

[3] J. Aczél, Gy. Maksa, Zs. Páles, Solution of a functional equation arising in an axiomatization of binary gambles, to appear in Proc. Amer. Math. Soc.

[4] J. Aczél, Gy. Maksa, Zs. Páles, Solutions to a functional equation arising from different ways of measuring utility, J. Math. Anal. Appl. 233(1999), 740-748.

[5] F. Bernstein, G. Doetsch, Zur Theorie der konvexen Funktionen, Math. Ann. 76(1915), 514-526.

[6] Z. Daróczy, A. Járai, On the measurable solution of a functional equation arising in information theory, Acta Math. Acad. Sci. Hung. 34(1979), 105-116.

[7] A. Gilányi, Zs. Páles, Remark 10, Report of Meeting, to appear in Rocznik Nauk.-Dydakt. Prace Math.

[8] A. Járai, On measurable solutions of functional equations, Publ. Math. Debrecen 26(1979), 17-35.

[9] A. Járai, Regularity properties of functional equations, Aequationes Math. 25(1982), 52-66.

[10] A. Járai, Regularity Properties of Functional Equations, Leaflets in Mathematics, Janus Pannonius University, Pécs, 1996.

[11] A. Járai, W. Sander, A regularity theorem in information theory, Publ. Math. Debrecen 50(1997), 339-357.

[12] A. Járai, L. Székelyhidi, Regularization and general methods in the theory of functional equations, Aequationes Math. 52(1996), 10-29.

[13] M. Kuczma, An Introduction to the Theory of Functional Equations and Inequalities, Państwowe Wydawnictwo Naukowe, Uniwersytet Śląski, Warszawa, 1985.

[14] Gy. Maksa, A.A.J. Marley, Zs. Páles, On a functional equation arising from joint-receipt utility models, to appear in Aequationes Math.

[15] Gy. Maksa, A.A.J. Marley, Zs. Páles, A simple characterization of binary rank-dependent expected utility of gains (losses) with exponential-like utility, submitted for publication.

[16] Zs. Páles, Remark 11 (to Problem 3 of J. Aczél), Report of Meeting, Aequationes Math. 56(1998), 306-307.

[17] Zs. Páles, Remark 23 (Solution to a Problem of J. Aczél), Report of Meeting, Aequationes Math. 56(1998), 312-314.

[18] Zs. Páles, Újabb módszerek a függvényegyenletek és függvényegyenlőtlenségek elméletében, MTA Doktori értekezés, [New Methods in the Theory of Functional Equations and Inequalities, D.Sc. Thesis, (in Hungarian)], Debrecen, 1999.

[19] Zs. Páles, Solution and regularity theory of composite functional equations, Abstract, Report of Meeting, to appear in Rocznik Nauk.-Dydakt. Prace Math.

Attila Gilányi, Institute of Mathematics and Informatics, University of DebreCen, 4010 Debrecen, Pf. 12, Hungary

E-mail address: gil@@math.klte.hu

Zsolt PÁles, Institute of Mathematics and Informatics, University of Debrecen, 4010 Debrecen, Pf. 12, Hungary

E-mail address: pales@@math.klte.hu 